\title{
Original
}

\section{Geomorphometrics of Tooth Size and Arch Dimension Analysis by Conventional Digital Caliper and Digital Stereomicroscope to Establish Standard Norms for the Pakistani Population}

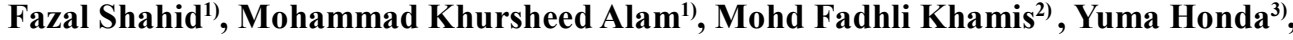 \\ Yoshihiko Sugita ${ }^{3)}$ and Hatsuhiko Maeda ${ }^{3)}$ \\ 1) Orthodontic Unit, School of Dental Science, Universiti Sains Malaysia, Kelantan, Malaysia \\ 2) Forensic Dentistry Unit, School of Dental Science, Universiti Sains Malaysia, Kelantan, Malaysia \\ ${ }^{3)}$ Department of Oral Pathology, School of Dentistry, Aichi Gakuin University, Nagoya, Japan \\ (Accepted for publication, February 17, 2015)
}

\begin{abstract}
In order to establish standard norms for the Pakistani population, we investigated the geomorphometrics of tooth size and arch dimension using a conventional digital caliper (DC) and a digital stereomicroscope (SM). The sample consisted of 128 subjects ranging in age from 18 to 24 years. A total of 44,155 variables were measured. Dental models of each subject for maxillary and mandibular arches were scanned via Hirox digital stereomicroscope for the fabrication of the digital models, and the geomorphometrics of tooth size and arch dimensions were measured via SM scanned digital models. All the measurements were also carried out via DC on plaster dental models. Sex differences and changes associated with methods were assessed, and interrelationships between different variables were explored within the study group. For the data obtained by DC and SM techniques, the men had significantly larger arch dimensions and geomorphometrics of tooth size than the women did. There were no significant disparities observed between the techniques. The developed norms for the mesiodistal, buccolingual, diagonal tooth sizes and arch dimension had significantly greater values for males in relation to females (*pd" 0.05 to ***pd" 0.001 ). This study has established a new reference database of tooth size and arch dimensions via both DC and digital SM for first time on Pakistani population. These norms will be helpful for clinical treatment planning in dentistry. Contemporaneously, the norms will be of great value to forensic dentists and dental anthropologists for making comparisons within and between different populations.
\end{abstract}

Key words: Mesiodistal; Buccolingual; Diagonal; Geomorphometric tooth size; Dental arch dimensions; Pakistani; Digital dental models

\section{Introduction}

Malocclusion is a common dilemma in all populations. Although the nature of malocclusion varies in different population, but teeth size and arch length inconsistency are considered to be an important etiological factors ${ }^{1}$. The teeth size and arch dimension are of preponderant importance in the various dental specialties such as Orthodontics, Prosthodontics, Forensic Dentistry and Dental Anthropology.

Nature has given an ideal balance between the maxillary and mandibular teeth size that should be attained for the ideal occlusion and aesthetics, especially in relation to the finishing phase in orthodontics ${ }^{2-4}$. There is obvious population variation in the pattern

Correspondence to: Dr. Mohammad Khursheed Alam, Orthodontic Unit, School of Dental Science, Universiti Sains Malaysia, Kubang Kerian, 16150, Kota Bharu, Kelantan, Malaysia; Phone: +60142926987 ; Email: dralam@gmail.com,dralam@usm.my and magnitude of sexual dimorphism ${ }^{5-7)}$. Teeth in relation to sexual dimorphism have been of prodigious importance to anthropologists and forensic odontologists as well as the focus of many studies for gender assessment ${ }^{8,9)}$.

Conventional caliper was used by researchers to investigate tooth dimension, to identify the sexual disparities through mesiodistal $1^{4,10,11,12)}$, buccolingual ${ }^{9,10,11,12)}$ and diagonal crown ${ }^{13)}$ diameters of teeth. Geomorphometrics is the quantitative approach that refer to the morphology of an entity depends on landmarks. This technique resolves numerous problems accompanying with out-of-date methods of measurements ${ }^{14}$.

The aim of the current study was to investigate the geomorphometrics of teeth size and arch dimension analysis by conventional digital caliper (DC) and a novel techniques by digital stereomicroscope (SM). The geomorphometrics include the mesiodistal, buccolingual, diagonal tooth diameters and arch 
dimension of the maxilla and mandible, correspondingly to reconnoiter the following objectives for the first time in Pakistani population.

- To determine the significant differences in measurements obtained via DC and SM.

- To develop tooth geomorphometric norms using-

$>$ Mesiodistal crown diameter

$>$ Buccolingual crown diameter

$>$ Diagonal crown diameter

- To develop arch dimension norms of maxilla and mandible using DC and SM

- To compare the mesiodistal, buccolingual and diagonal crown diameters of the maxillary and mandibular arch between right and left sides

- To evaluate the amount of sexual dimorphism

\section{Materials and Methods}

\section{Subjects}

All participants provided their written informed consent. Ethics approval was obtained from the Ethics Committee of the Universiti Sains Malaysia (USM/JEPeM/140376). This study was designed and conducted according to the guidelines of strengthening the Reporting of Observational studies in Epidemiology (STROBE), and we applied the STROBE specification in this manuscript ${ }^{15)}$.

\section{Sample size calculation}

The sample size was calculated at the power of $80 \%$, alpha 0.05 , estimated standard deviation $0.60 \mathrm{~mm}^{16)}$, mean difference, biologically meaningful $0.3 \mathrm{~mm}$, and equal sample size were taken by utilizing the PS software ${ }^{17}$. The calculated sample size was 64 males and 64 females $(m=1)$.Total 44,155 variables were measured with the following inclusion and exclusion criteria.

\section{Inclusion criteria}

- Subjects were from the Pakistani population determined via interviews with mutually paternities and ancestors without any multi-ethnic nuptial.

- Age ranges from 18 to 24

- Subjects with all sound erupted permanent teeth (except third molar), with no history of previous orthodontic treatment.

- Class I incisor relationship according to British Standards Institute

- Class I molar and canine relationship

- No crowding, cross bite and spacing

- Straight profile (identify by examining the profile view)

- no craniofacial anomalies

\section{Exclusion criteria}

- Inter proximal caries or restoration

- Missing or supernumerary teeth
- Abnormal size or morphology of teeth

- Teeth wear that affect the tooth size measurement

- Damage casts

Oral examinations were carried out with a vigilant assortment of participants. Cross-examination of subjects was performed to minimize selection bias and error; experienced orthodontist and dentists participated throughout the screening sessions.

Dental impressions of the upper and lower arches of each subject were taken with alginate impression material (Zhermack Orthoprint alginate ISO 1563 -ADA 18 Italy) and poured with dental stone (Type III hard plaster quick stone China). The impressions were poured with dental stone within an hour. The set dental casts were disinfected by immersion with $0.5 \%$ sodium hypochlorite for 10 minutes and labeled.

\section{Measurement of tooth size and arch dimensions}

These measurements were carried out for tooth size and arch dimension via two methods as below:

\section{Measurement via SM technique}

Dental models of each subject for maxillary and mandibular arches were scanned via Hirox digital stereomicroscope (HIROX KH7700 Japan) for the fabrication of the digital models (Fig. 1). Geomorphometrics of tooth size and arch dimensions were carried out via SM scanned digital models. SM was proven as valid ${ }^{18)}$ and reliable tool for such measurements with the accuracy of $0.1 \times 10^{-6}$, while manual and digital sliding caliper can measure with the accuracy $0.01 \mathrm{~mm}$, respectively. The acquisitions of measurements for teeth size and arch dimensions were as the following:

\section{Teeth size measurement for maxillary and mandibular arches} Mesiodistal crown diameter (Fig. 2a)

The mesiodistal crown diameter of the tooth was measured from anatomical contact of one tooth to another on occlusal side perpendicular to the long axis of the teeth ${ }^{1,4,11,19,20)}$.

Buccolingual crown diameters (Fig. 2b)

Buccolingual crown diameters recorded as the greatest distance between buccal/labial and lingual surfaces perpendicular to the occlusal plane $\mathrm{e}^{11,21)}$

Diagonal diameters (Fig. 2c)

The measurements were taken with morphometric points through the largest distance between the mesiobuccal to distolingual (MBDL) and distobuccal to the mesiolingual (DBML) points of the crown ${ }^{13)}$.

\section{Arch dimension for maxillary and mandibular arches ${ }^{18,19)}$} Arch perimeter (Fig. 3 a1)

The sum of fragmental lines from right and left side of the arch (these segments are starting from the distal contact of the 
Fazal Shahid et al.: Geomorphometrics of Tooth Size and Arch Dimension

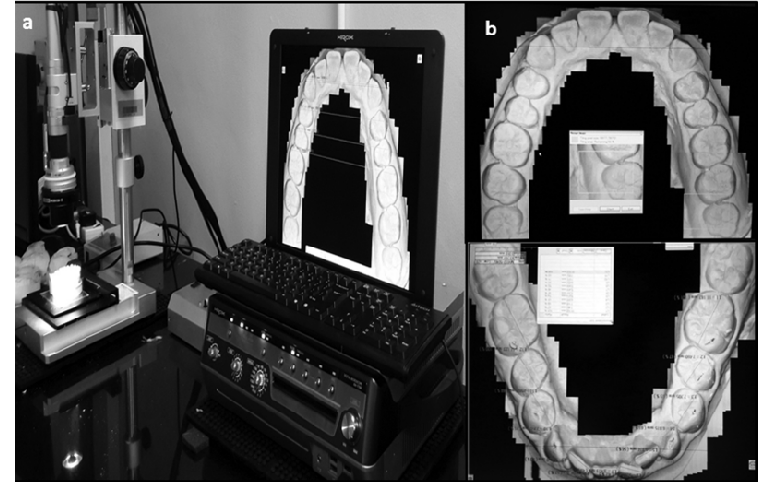

Figure 1. Fabrication of the digital models (a) Hirox digital stereomicroscope (b) digital models of maxilla and mandible

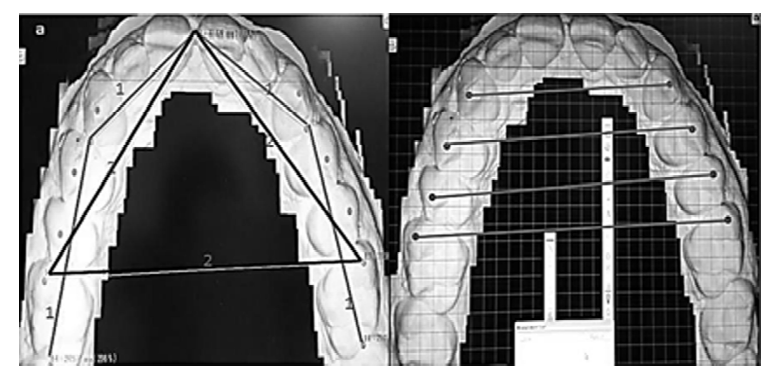

Figure 3. Arch dimension measurements (a1) arch perimeter (a2) arch length (b) Arch width measurements

molar to the mesial contact point of the canine and continue to the contact point of the central incisors).

Arch Length (Fig. 3 a2)

Transverse line connecting between three points, as from the mesio-buccal cusp tip to the mesio-buccal cusp tip of the opposite side of first permanent molar and the line then protracted from both side anteriorly to the centre point between the central incisors for both the maxillary and mandibular arches.

Arch width (Fig. 3b)

- Inter canine widths were obtained from cusp tip of one side to the opposing side cusp tip.

- Inter 1st premolar and 2nd premolar widths were obtained from buccal cusp tip to the buccal cusp tip of the contralateral side.

- Inter first molar width of the maxillary and mandibular arches were obtained from the mesiobuccal cusp tip of right side to the left side.

\section{Measurement via DC technique}

The measurements for teeth size and arch dimension were carried out via DC (Mitutoyo, Japan).

Teeth size

Teeth size measurements were obtained for the mesiodistal ${ }^{4,9-}$ ${ }^{11)}$ (Fig. 4a), buccolingual 9-12) (Fig. 4b), and diagonal ${ }^{13)}$ (Fig. 5) crown diameters.

\section{Arch dimension}

Maxillary and mandibular linear arch dimensions were

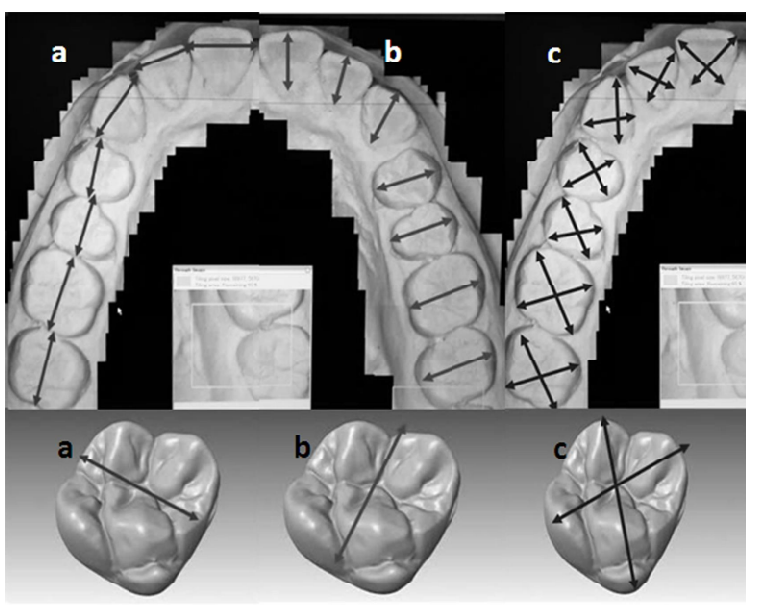

Figure 2. Measurement of tooth size (a) mesiodistal crown diameter (b) buccolingual crown diameter (c) diagonal crown diameter

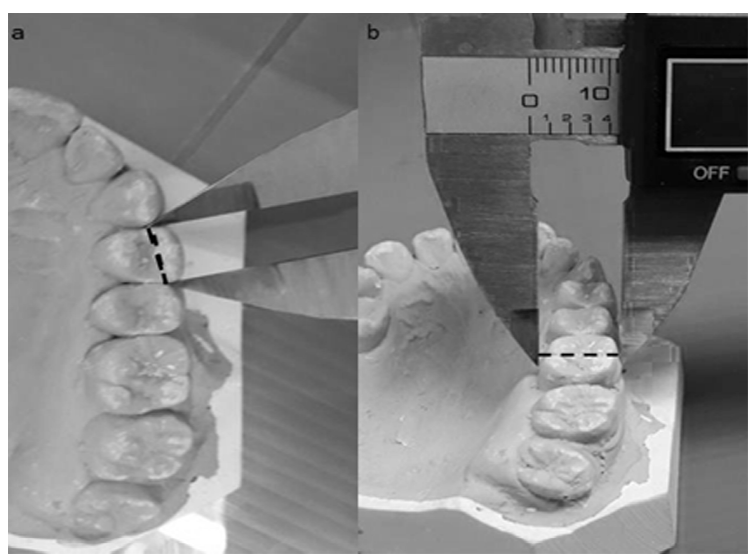

Figure 4. DC measurements (a) Mesiodistal crown diameter (b) Buccolingual crown diameter

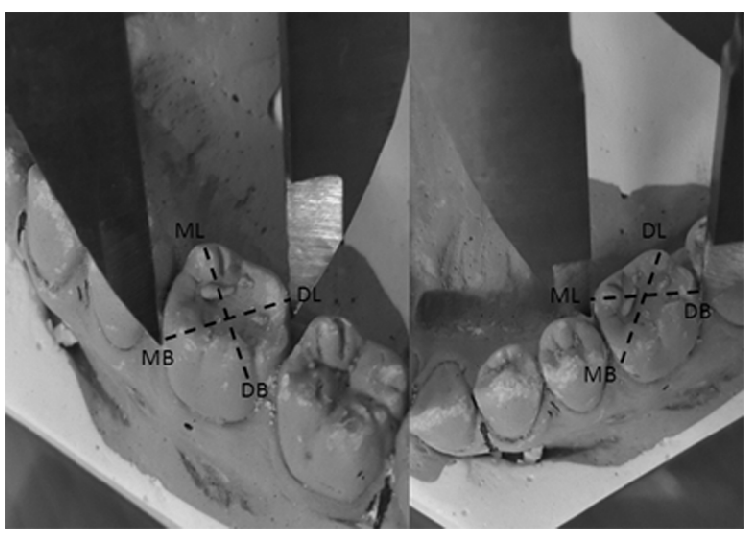

Figure 5. Diagonal crown diameter via DC

measured for the arch perimeter (Fig. 6a), arch length (Fig. 6b), and arch widths (Fig. 7) inter canine, inter first and second premolars, and inter first molar area.

\section{Error study}

$20 \%$ of dental cast were randomly selected for intra observer errors. The time interval between the first and second reading 


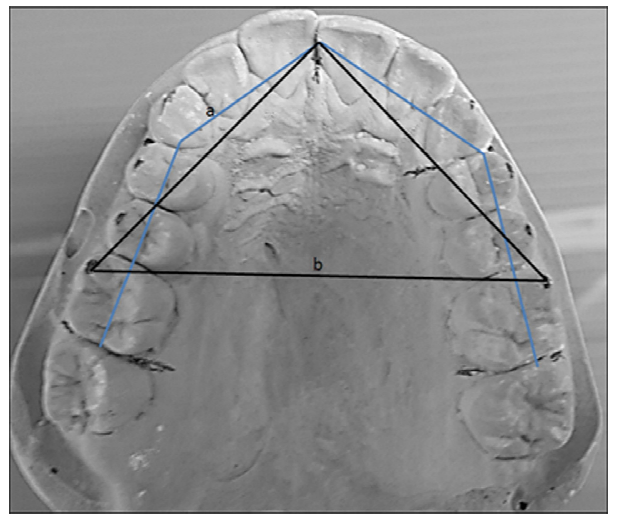

Figure 6. Arch dimension via DC (a) Arch perimeter (b) Arch length

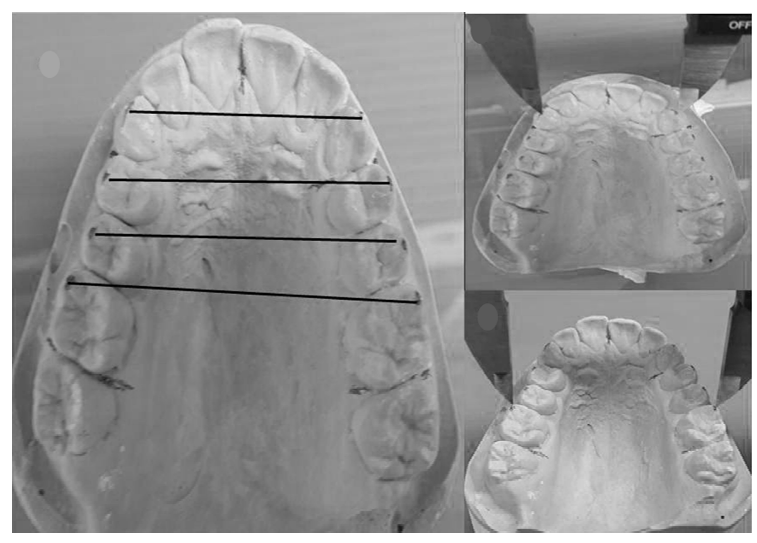

Figure 7. Arch width measurements via DC

Table 1. DC vs. SM for mesiodistal crown diameters.

\begin{tabular}{lllllllll}
\hline Variables* & DC & & SM & & Mean diff. & $\mathbf{9 5 \%}$ CI & & $\boldsymbol{p}$ \\
\hline & Mean & SD & Mean & SD & & Lower & Upper & \\
$\mathbf{1 1}$ & & & & & & & & \\
$\mathbf{1 2}$ & 6.58 & 0.49 & 8.59 & 0.50 & -0.01 & -0.13 & 0.10 & .831 \\
$\mathbf{1 3}$ & 7.84 & 0.52 & 6.88 & 0.49 & 0.00 & -0.12 & 0.12 & .965 \\
$\mathbf{1 4}$ & 6.95 & 0.48 & 7.85 & 0.48 & -0.01 & -0.13 & 0.10 & .831 \\
$\mathbf{1 5}$ & 6.69 & 0.48 & 6.96 & 0.44 & -0.01 & -0.11 & 0.10 & .885 \\
$\mathbf{1 6}$ & 10.08 & 0.61 & 10.09 & 0.60 & -0.01 & -0.16 & 0.13 & .871 \\
$\mathbf{1 7}$ & 9.27 & 0.58 & 9.27 & 0.59 & -0.01 & -0.15 & 0.13 & .897 \\
$\mathbf{2 1}$ & 8.53 & 0.49 & 8.56 & 0.50 & -0.04 & -0.15 & 0.08 & .553 \\
$\mathbf{2 2}$ & 6.80 & 0.50 & 6.83 & 0.50 & -0.03 & -0.15 & 0.09 & .598 \\
$\mathbf{2 3}$ & 7.78 & 0.48 & 7.81 & 0.48 & -0.04 & -0.15 & 0.08 & .526 \\
$\mathbf{2 4}$ & 6.98 & 0.46 & 7.01 & 0.45 & -0.03 & -0.14 & 0.08 & .586 \\
$\mathbf{2 5}$ & 6.65 & 0.49 & 6.68 & 0.48 & -0.03 & -0.14 & 0.09 & .658 \\
$\mathbf{2 6}$ & 10.00 & 0.57 & 10.04 & 0.58 & -0.04 & -0.18 & 0.10 & .568 \\
$\mathbf{2 7}$ & 9.28 & 0.55 & 9.31 & 0.56 & -0.03 & -0.16 & 0.10 & .673 \\
$\mathbf{3 1}$ & 5.48 & 0.37 & 5.50 & 0.36 & -0.03 & -0.11 & 0.06 & .525 \\
$\mathbf{3 2}$ & 5.97 & 0.40 & 6.00 & 0.39 & -0.03 & -0.13 & 0.06 & .502 \\
$\mathbf{3 3}$ & 6.89 & 0.46 & 6.95 & 0.45 & -0.05 & -0.16 & 0.06 & .341 \\
$\mathbf{3 4}$ & 6.99 & 0.42 & 7.03 & 0.42 & -0.03 & -0.13 & 0.06 & .494 \\
$\mathbf{3 5}$ & 6.98 & 0.47 & 7.02 & 0.46 & -0.03 & -0.14 & 0.08 & .545 \\
$\mathbf{3 6}$ & 11.09 & 0.75 & 11.12 & 0.74 & -0.02 & -0.20 & 0.15 & .791 \\
$\mathbf{3 7}$ & 9.96 & 0.82 & 10.03 & 0.69 & -0.07 & -0.25 & 0.11 & .465 \\
$\mathbf{4 1}$ & 5.47 & 0.37 & 5.48 & 0.37 & -0.01 & -0.10 & 0.08 & .829 \\
$\mathbf{4 2}$ & 5.94 & 0.33 & 5.95 & 0.34 & -0.01 & -0.09 & 0.07 & .884 \\
$\mathbf{4 3}$ & 6.87 & 0.43 & 6.84 & 0.67 & 0.03 & -0.10 & 0.16 & .657 \\
$\mathbf{4 4}$ & 7.02 & 0.43 & 7.03 & 0.42 & -0.01 & -0.11 & 0.09 & .888 \\
$\mathbf{4 5}$ & 6.97 & 0.46 & 6.99 & 0.49 & -0.02 & -0.13 & 0.10 & .786 \\
$\mathbf{4 6}$ & 11.15 & 0.73 & 11.15 & 0.73 & 0.00 & -0.18 & 0.17 & .960 \\
$\mathbf{4 7}$ & 10.04 & 0.70 & 10.05 & 0.70 & 0.00 & -0.17 & 0.16 & .956 \\
\hline
\end{tabular}

*FDI notation; DC, digital caliper; SM, hirox digital stereomicroscope; CI, confidence interval; SD, standard deviation; Mean diff., mean differences.

were approximately 2 weeks.

The method error was analyzed by the Dalhberg's formula:

$$
\mathrm{ME}=\left(\Sigma\left(\mathrm{x}_{1}-\mathrm{x}_{2}\right)^{2 / 2} 2(28)\right)^{1 / 2}
$$

Where $\mathrm{x}_{1}$ is the first measurement, $\mathrm{x}_{2}$ the second measurement and $n=1$ the number of sample repeated ${ }^{22}$.

\section{Statistical analyses}

Descriptive data were verified and analyzed statistically using IBM SPSS Statistics Version 22.0 with significance level set at
$5 \%(\mathrm{P}<0.05)$. An independent samples t-test was used to evaluate differences in sex. The paired sample $t$ test was used to determine differences in teeth size between the right and left side of the maxillary and mandibular arches, and difference in techniques between DC and SM.

\section{Results}

\section{Error of the method for DC and SM measurements}

The method error was analyzed by the Dalhberg's formula, 
Fazal Shahid et al.: Geomorphometrics of Tooth Size and Arch Dimension

Table 2. DC vs. SM for buccolingual crown diameters.

\begin{tabular}{|c|c|c|c|c|c|c|c|c|}
\hline \multicolumn{2}{|c|}{ Variables* DC } & \multicolumn{3}{|c|}{ SM } & \multirow{2}{*}{$\begin{array}{l}\text { Mean diff. } \\
\text { Lower }\end{array}$} & \multicolumn{2}{|l|}{$95 \%$ CI } & \multirow[t]{2}{*}{$p$} \\
\hline Mean & SD & Mean & SD & & & Upper & & \\
\hline 11 & 6.55 & 0.62 & 6.55 & 0.62 & 0.09 & -0.15 & 0.15 & .990 \\
\hline 12 & 5.63 & 0.57 & 5.65 & 0.56 & -0.02 & -0.16 & 0.11 & .735 \\
\hline 13 & 7.52 & 0.61 & 7.55 & 0.59 & -0.03 & -0.17 & 0.11 & .689 \\
\hline 14 & 8.77 & 0.56 & 8.80 & 0.54 & -0.03 & -0.16 & 0.10 & .668 \\
\hline 15 & 8.88 & 0.56 & 8.89 & 0.55 & -0.01 & -0.15 & 0.12 & .839 \\
\hline 16 & 10.80 & 0.58 & 10.81 & 0.58 & -0.01 & -0.15 & 0.13 & .901 \\
\hline 17 & 10.29 & 0.72 & 10.33 & 0.70 & -0.04 & -0.21 & 0.13 & .649 \\
\hline 21 & 6.56 & 0.61 & 6.56 & 0.61 & 0.00 & -0.14 & 0.14 & .990 \\
\hline 22 & 5.63 & 0.56 & 5.65 & 0.55 & -0.02 & -0.16 & 0.11 & .732 \\
\hline 23 & 7.50 & 0.61 & 7.53 & 0.59 & -0.03 & -0.17 & 0.12 & .710 \\
\hline 24 & 8.78 & 0.57 & 8.81 & 0.55 & -0.03 & -0.16 & 0.10 & .657 \\
\hline 25 & 8.88 & 0.55 & 8.89 & 0.55 & -0.01 & -0.14 & 0.12 & .845 \\
\hline 26 & 10.80 & 0.58 & 10.81 & 0.58 & -0.01 & -0.15 & 0.13 & .881 \\
\hline 27 & 10.21 & 0.73 & 10.26 & 0.72 & -0.05 & -0.22 & 0.13 & .593 \\
\hline 31 & 5.33 & 0.47 & 5.31 & 0.47 & 0.01 & -0.10 & 0.12 & .843 \\
\hline 32 & 5.46 & 0.54 & 5.46 & 0.54 & -0.01 & -0.13 & 0.12 & .927 \\
\hline 33 & 6.59 & 0.58 & 6.58 & 0.59 & 0.01 & -0.13 & 0.15 & .873 \\
\hline 34 & 7.34 & 0.50 & 7.34 & 0.51 & 0.00 & -0.12 & 0.12 & .984 \\
\hline 35 & 7.93 & 0.53 & 7.91 & 0.55 & 0.02 & -0.10 & 0.15 & .727 \\
\hline 36 & 10.18 & 0.54 & 10.18 & 0.55 & 0.00 & -0.13 & 0.13 & .994 \\
\hline 37 & 9.64 & 0.63 & 9.67 & 0.62 & -0.03 & -0.17 & 0.12 & .737 \\
\hline 41 & 5.32 & 0.47 & 5.31 & 0.47 & 0.01 & -0.10 & 0.12 & .815 \\
\hline 42 & 5.44 & 0.53 & 5.45 & 0.53 & -0.01 & -0.13 & 0.12 & .913 \\
\hline 43 & 6.59 & 0.54 & 6.58 & 0.55 & 0.02 & -0.11 & 0.14 & .816 \\
\hline 44 & 7.34 & 0.50 & 7.34 & 0.51 & 0.00 & -0.12 & 0.12 & .989 \\
\hline 45 & 7.93 & 0.51 & 7.90 & 0.54 & 0.03 & -0.10 & 0.15 & .692 \\
\hline 46 & 10.19 & 0.51 & 10.19 & 0.52 & 0.00 & -0.12 & 0.12 & .981 \\
\hline 47 & 9.55 & 0.94 & 9.66 & 0.51 & -0.11 & -0.29 & 0.07 & .239 \\
\hline
\end{tabular}

*FDI notation; DC, digital caliper; SM, hirox digital stereomicroscope; CI, confidence interval; SD, standard deviation; Mean diff., mean differences.

Table 3. DC vs. SM for maxillary arch diagonal crown diameters.

\begin{tabular}{|c|c|c|c|c|c|c|c|c|}
\hline Variables* & DC & & SM & & Mean & $95 \%$ & & $P$ \\
\hline & Mean & SD & Mean & SD & & Lower & Upper & \\
\hline 11 MxDDP & 8.21 & 0.49 & 8.22 & 0.49 & -0.02 & -0.13 & 0.10 & .764 \\
\hline 11MxDMP & 8.22 & 0.51 & 8.24 & 0.50 & -0.02 & -0.14 & 0.10 & .766 \\
\hline 12 MxDDP & 6.68 & 0.50 & 6.68 & 0.50 & 0.00 & -0.12 & 0.12 & :998 \\
\hline 12 MxDMP & 6.61 & 0.54 & 6.63 & 0.53 & -0.01 & -0.14 & 0.11 & .830 \\
\hline 13MxDDP & 7.52 & 0.42 & 7.54 & 0.40 & -0.03 & -0.12 & 0.07 & .610 \\
\hline 13MxDMP & 7.68 & 0.47 & 7.71 & 0.48 & -0.02 & -0.13 & 0.09 & .719 \\
\hline 14 MxDDP & 8.23 & 0.57 & 8.25 & 0.57 & -0.02 & -0.15 & 0.12 & .809 \\
\hline 14 MxDMP & 8.03 & 0.53 & 8.02 & 0.54 & 0.01 & -0.12 & 0.14 & .895 \\
\hline 15 MxDDP & 8.16 & 0.59 & 8.18 & 0.58 & -0.02 & -0.16 & 0.12 & .771 \\
\hline 15 MxDMP & 8.14 & 0.58 & 8.14 & 0.58 & 0.00 & -0.13 & 0.14 & .965 \\
\hline 16 MxDDP & 10.87 & 0.60 & 10.88 & 0.61 & -0.01 & -0.15 & 0.14 & .919 \\
\hline 16 MxDMP & 12.32 & 0.61 & 12.30 & 0.64 & 0.02 & -0.13 & 0.17 & .806 \\
\hline 17 MxDDP & 9.60 & 0.82 & 9.63 & 0.82 & -0.03 & -0.22 & 0.16 & .765 \\
\hline 17MxDMP & 10.83 & 0.98 & 10.83 & 0.97 & 0.00 & -0.23 & 0.23 & .974 \\
\hline 21 MxDDP & 8.18 & 0.55 & 8.20 & 0.55 & -0.02 & -0.15 & 0.11 & .789 \\
\hline 21 MxDMP & 8.20 & 0.57 & 8.21 & 0.57 & -0.02 & -0.15 & 0.12 & .806 \\
\hline 22 MxDDP & 6.68 & 0.50 & 6.68 & 0.50 & 0.00 & -0.12 & 0.12 & .980 \\
\hline 22 MxDMP & 6.61 & 0.54 & 6.62 & 0.54 & -0.02 & -0.14 & 0.11 & .812 \\
\hline 23 MxDDP & 7.51 & 0.43 & 7.54 & 0.41 & -0.03 & -0.13 & 0.07 & .590 \\
\hline 23 MxDMP & 7.68 & 0.47 & 7.70 & 0.48 & -0.02 & -0.13 & 0.09 & .705 \\
\hline 24 MxDDP & 8.22 & 0.59 & 8.24 & 0.59 & -0.02 & -0.16 & 0.12 & .828 \\
\hline 24 MxDMP & 8.02 & 0.54 & 8.01 & 0.54 & 0.01 & -0.12 & 0.14 & .878 \\
\hline 25 MxDDP & 8.15 & 0.59 & 8.17 & 0.59 & -0.02 & -0.16 & 0.12 & .777 \\
\hline 25 MxDMP & 8.13 & 0.58 & 8.13 & 0.58 & 0.00 & -0.13 & 0.14 & .946 \\
\hline 26 MxDDP & 10.87 & 0.60 & 10.88 & 0.61 & -0.01 & -0.15 & 0.14 & .932 \\
\hline 26MxDMP & 12.31 & 0.61 & 12.32 & 0.61 & 0.00 & -0.15 & 0.14 & .971 \\
\hline 27 MxDDP & 9.62 & 0.82 & 9.60 & 1.05 & 0.01 & -0.21 & 0.24 & .901 \\
\hline 27 MxDMP & 10.82 & 0.98 & 10.82 & 0.97 & 0.00 & -0.23 & 0.23 & .979 \\
\hline
\end{tabular}

*FDI notation; DC, digital caliper; SM, hirox digital stereomicroscope; MxDDP, maxillary diagonal distopalatal diameter; MxDMP, maxillary diagonal mesiopalatal diameter; CI, confidence interval; SD, standard deviation; Mean diff., mean differences. 
J.Hard Tissue Biology Vol. 24(2):155 -168, 2015

Table 4. DC vs. SM for the mandibular arch diagonal crown diameters.

\begin{tabular}{lllllllll}
\hline Variables* & DC & & SM & \multicolumn{3}{c}{ Mean diff. 95\% CI } & P \\
\hline 31 MnDDL & Mean & SD & Mean & SD & & Lower & Upper & \\
31 MnDML & 5.26 & 0.45 & 5.30 & 0.43 & -0.04 & -0.15 & 0.06 & .447 \\
32 MnDDL & 5.74 & 0.45 & 5.21 & 0.45 & -0.05 & -0.15 & 0.06 & .406 \\
32 MnDML & 5.69 & 0.43 & 5.76 & 0.43 & -0.02 & -0.12 & 0.08 & .688 \\
33 MnDDL & 6.53 & 0.45 & 5.72 & 0.44 & -0.03 & -0.13 & 0.08 & .604 \\
33 MnDML & 6.75 & 0.46 & 6.76 & 0.42 & -0.03 & -0.14 & 0.07 & .537 \\
34 MnDDL & 6.87 & 0.43 & 6.88 & 0.45 & -0.01 & -0.12 & 0.10 & .821 \\
34 MnDML & 7.18 & 0.49 & 7.19 & 0.48 & -0.01 & -0.11 & 0.09 & .800 \\
35 MnDDL & 7.52 & 0.51 & 7.56 & 0.52 & -0.03 & -0.13 & 0.10 & .782 \\
35 MnDML & 7.68 & 0.59 & 7.73 & 0.59 & -0.06 & -0.20 & 0.09 & .595 \\
36 MnDDL & 11.23 & 0.61 & 11.26 & 0.59 & -0.02 & -0.17 & 0.12 & .745 \\
36 MnDML & 11.70 & 0.60 & 11.73 & 0.58 & -0.03 & -0.17 & 0.11 & .644 \\
37 MnDDL & 10.69 & 0.73 & 10.72 & 0.71 & -0.04 & -0.21 & 0.14 & .685 \\
37 MnDML & 10.83 & 0.67 & 10.85 & 0.67 & -0.03 & -0.18 & 0.13 & .750 \\
41 MnDDL & 5.26 & 0.45 & 5.30 & 0.43 & -0.04 & -0.15 & 0.06 & .448 \\
41 MnDML & 5.17 & 0.45 & 5.21 & 0.45 & -0.05 & -0.15 & 0.06 & .404 \\
42 MnDDL & 5.74 & 0.43 & 5.76 & 0.43 & -0.02 & -0.12 & 0.08 & .691 \\
42 MnDML & 5.69 & 0.45 & 5.72 & 0.44 & -0.03 & -0.13 & 0.08 & .604 \\
43 MnDDL & 6.53 & 0.45 & 6.56 & 0.42 & -0.03 & -0.14 & 0.07 & .524 \\
43 MnDML & 6.75 & 0.46 & 6.76 & 0.45 & -0.01 & -0.12 & 0.10 & .848 \\
44 MnDDL & 6.87 & 0.43 & 6.88 & 0.43 & -0.01 & -0.11 & 0.09 & .818 \\
44 MnDML & 7.18 & 0.49 & 7.19 & 0.48 & -0.02 & -0.13 & 0.10 & .785 \\
45 MnDDL & 7.52 & 0.51 & 7.56 & 0.52 & -0.03 & -0.16 & 0.09 & .583 \\
45 MnDML & 7.68 & 0.59 & 7.73 & 0.59 & -0.06 & -0.20 & 0.08 & .406 \\
46 MnDDL & 11.23 & 0.61 & 11.25 & 0.60 & -0.02 & -0.16 & 0.13 & .824 \\
46 MnDML & 11.75 & 0.92 & 11.73 & 0.58 & 0.03 & -0.16 & 0.21 & .784 \\
47 MnDDL & 10.69 & 0.73 & 10.72 & 0.72 & -0.03 & -0.20 & 0.14 & .736 \\
47 MnDML & 10.83 & 0.67 & 10.85 & 0.67 & -0.02 & -0.18 & 0.14 & .784 \\
\hline
\end{tabular}

*FDI notation; DC, digital caliper; SM, hirox digital stereomicroscope; MnDDL, mandibular diagonal distolingual diameter; MnDML, mandibular diagonal mesiolingual diameter; CI, confidence interval; SD, standard deviation; Mean diff., mean differences.

Table 5. DC vs. SM for the arch dimensions

\begin{tabular}{lllllllll}
\hline Variables & DC & \multicolumn{3}{c}{ SM } & \multicolumn{3}{c}{ Mean diff. 95\% CI } & \multicolumn{1}{c}{$\boldsymbol{P}$} \\
\hline UAP & Mean & SD & Mean & SD & & Lower & Upper & \\
UAL & 96.24 & 4.54 & 96.28 & 4.66 & -0.05 & -1.14 & 1.04 & .929 \\
UICW & 128.95 & 5.14 & 129.09 & 5.41 & -0.14 & -1.39 & 1.11 & .828 \\
UI1PW & 35.18 & 2.30 & 35.19 & 2.30 & -0.01 & -0.56 & 0.53 & .958 \\
UI2PW & 42.73 & 2.65 & 42.73 & 2.65 & 0.00 & -0.63 & 0.63 & .993 \\
UIMW & 53.08 & 2.56 & 48.08 & 2.56 & 0.00 & -0.61 & 0.61 & .998 \\
LAP & 89.35 & 2.62 & 53.13 & 2.63 & 0.00 & -0.62 & 0.62 & .996 \\
LAL & 111.58 & 5.59 & 89.39 & 4.65 & -0.04 & -1.14 & 1.06 & .938 \\
LICW & 26.67 & 1.95 & 26.67 & 1.95 & 0.00 & -0.47 & 0.46 & .990 \\
LI1PW & 35.23 & 2.33 & 35.24 & 2.33 & 0.00 & -0.56 & 0.55 & .994 \\
LI2PW & 41.15 & 2.59 & 41.16 & 2.59 & 0.00 & -0.62 & 0.61 & .993 \\
LIMW & 45.98 & 2.52 & 45.98 & 2.52 & 0.00 & -0.60 & 0.59 & .993 \\
\hline
\end{tabular}

DC, digital caliper; SM, hirox digital stereomicroscope; UAP, upper arch perimeter; UAL, upper arch length; UICW, upper inter canine width; UI1PW, upper inter first premolar width; UI2PW, upper inter second premolar width; UIMW, upper inter molar width; LAP, lower arch perimeter; LAL, lower arch length; LICW, Lower inter canine width; LI1 PW, Lower inter first premolar width; LI2PW, Lower inter second premolar width; LIMW, Lower inter molar width; CI, confidence interval; SD, standard deviation; Mean diff., mean differences.

showed the values of $0.006,0.007,0.004,0.0005$ and 0.033 for mesiodistal, buccolingual, and diagonal maxillary, diagonal mandibular and arch dimension respectively via DC. Whereas the SM showed $0.005,0.004,0.0109,0.012,0.001$ for the above variables respectively.

Inter measurement disparities between DC and SM techniques Tables 1, 2, 3, 4 and 5 show the comparison of tooth sizes and 
Fazal Shahid et al.: Geomorphometrics of Tooth Size and Arch Dimension

Table 6. Mesiodistal crown diameters measurement via DC, Norms and Inter sex disparities.

\begin{tabular}{|c|c|c|c|c|c|c|c|c|c|}
\hline Variables* & Male & & Fema & & Mean diff. & $95 \%$ CI & & $\boldsymbol{P}$ & \\
\hline & Mean & SD & Mean & SD & & Lower & Upper & & \\
\hline 11 & 8.71 & 0.44 & 8.43 & 0.50 & 0.28 & 0.13 & 0.44 & .001 & $* * *$ \\
\hline 12 & 6.96 & 0.51 & 6.77 & 0.51 & 0.20 & 0.02 & 0.37 & .026 & $* *$ \\
\hline 13 & 7.97 & 0.45 & 7.70 & 0.48 & 0.27 & 0.11 & 0.43 & .001 & $* * *$ \\
\hline 14 & 7.03 & 0.46 & 6.87 & 0.45 & 0.16 & 0.01 & 0.32 & .036 & * \\
\hline 15 & 6.74 & 0.46 & 6.63 & 0.50 & 0.11 & -0.05 & 0.27 & .171 & \\
\hline 16 & 10.18 & 0.68 & 9.96 & 0.49 & 0.22 & 0.02 & 0.43 & .031 & $* *$ \\
\hline 17 & 9.44 & 0.58 & 9.06 & 0.52 & 0.38 & 0.20 & 0.57 & .001 & $* * *$ \\
\hline 21 & 8.69 & 0.45 & 8.33 & 0.46 & 0.36 & 0.21 & 0.51 & .001 & $* * *$ \\
\hline 22 & 6.88 & 0.50 & 6.70 & 0.48 & 0.17 & 0.01 & 0.34 & .040 & $*$ \\
\hline 23 & 7.91 & 0.44 & 7.62 & 0.48 & 0.29 & 0.14 & 0.44 & .001 & $* * *$ \\
\hline 24 & 7.06 & 0.47 & 6.90 & 0.44 & 0.16 & 0.01 & 0.31 & .041 & $*$ \\
\hline 25 & 6.72 & 0.46 & 6.56 & 0.51 & 0.16 & -0.01 & 0.32 & .058 & $*$ \\
\hline 26 & 10.11 & 0.61 & 9.87 & 0.50 & 0.24 & 0.05 & 0.43 & .014 & $* *$ \\
\hline 27 & 9.41 & 0.60 & 9.13 & 0.45 & 0.28 & 0.09 & 0.46 & .003 & $* *$ \\
\hline 31 & 5.48 & 0.35 & 5.47 & 0.39 & 0.01 & -0.11 & 0.14 & .859 & \\
\hline 32 & 6.03 & 0.41 & 5.91 & 0.38 & 0.12 & -0.01 & 0.25 & .076 & \\
\hline 33 & 7.02 & 0.40 & 6.74 & 0.48 & 0.28 & 0.13 & 0.43 & .001 & $* * *$ \\
\hline 34 & 7.02 & 0.45 & 6.97 & 0.39 & 0.05 & -0.09 & 0.20 & .457 & \\
\hline 35 & 7.04 & 0.45 & 6.92 & 0.49 & 0.12 & -0.04 & 0.28 & .138 & \\
\hline 36 & 11.21 & 0.75 & 10.96 & 0.74 & 0.25 & 0.00 & 0.50 & .051 & $*$ \\
\hline 37 & 10.15 & 0.80 & 9.75 & 0.79 & 0.40 & 0.13 & 0.67 & .004 & ** \\
\hline 41 & 5.51 & 0.34 & 5.44 & 0.39 & 0.07 & -0.05 & 0.19 & .269 & \\
\hline 42 & 6.01 & 0.36 & 5.86 & 0.29 & 0.15 & 0.04 & 0.26 & .009 & $* *$ \\
\hline 43 & 6.99 & 0.42 & 6.75 & 0.41 & 0.24 & 0.10 & 0.38 & .001 & $* * *$ \\
\hline 44 & 7.05 & 0.44 & 7.00 & 0.41 & 0.05 & -0.09 & 0.19 & .493 & \\
\hline 45 & 7.03 & 0.47 & 6.90 & 0.45 & 0.13 & -0.02 & 0.29 & .095 & \\
\hline 46 & 11.28 & 0.71 & 10.99 & 0.73 & 0.29 & 0.05 & 0.54 & .018 & $* *$ \\
\hline 47 & 10.21 & 0.70 & 9.85 & 0.65 & 0.37 & 0.14 & 0.60 & .002 & $* *$ \\
\hline
\end{tabular}

*FDI notation; DC, digital caliper; CI, confidence interval; SD, standard deviation; Mean diff., mean differences; $(* * *$ pd" 0.001$)$, $(* * p d " 0.01)$ and (*pd" 0.05).

Table 7. Buccolingual crown diameters measurement via DC, Norms and Inter sex disparities.

\begin{tabular}{|c|c|c|c|c|c|c|c|c|c|}
\hline \multicolumn{3}{|c|}{ Variables* Male } & \multicolumn{2}{|c|}{ Female } & \multicolumn{3}{|c|}{ Mean diff. 95\% CI } & \multicolumn{2}{|l|}{$p$} \\
\hline & Mean & SD & Mean & SD & & Lower & Upper & & \\
\hline 11 & 6.78 & 0.59 & 6.28 & 0.55 & 0.50 & 0.31 & 0.69 & .001 & $* * *$ \\
\hline 12 & 5.81 & 0.52 & 5.41 & 0.55 & 0.40 & 0.22 & 0.58 & .001 & $* * *$ \\
\hline 13 & 7.74 & 0.60 & 7.28 & 0.53 & 0.46 & 0.27 & 0.66 & .001 & $* * *$ \\
\hline 14 & 8.93 & 0.51 & 8.59 & 0.55 & 0.34 & 0.16 & 0.52 & .001 & $* * *$ \\
\hline 15 & 9.01 & 0.49 & 8.73 & 0.60 & 0.29 & 0.10 & 0.47 & .003 & $* *$ \\
\hline 16 & 10.95 & 0.50 & 10.63 & 0.62 & 0.33 & 0.14 & 0.52 & .001 & $* * *$ \\
\hline 17 & 10.36 & 0.65 & 10.22 & 0.79 & 0.14 & -0.10 & 0.38 & .263 & \\
\hline 21 & 6.79 & 0.56 & 6.29 & 0.54 & 0.50 & 0.32 & 0.69 & .001 & $* * *$ \\
\hline 22 & 5.80 & 0.55 & 5.43 & 0.52 & 0.37 & 0.19 & 0.55 & .001 & $* * *$ \\
\hline 23 & 7.73 & 0.55 & 7.23 & 0.57 & 0.50 & 0.31 & 0.69 & .001 & $* * *$ \\
\hline 24 & 8.91 & 0.54 & 8.62 & 0.57 & 0.28 & 0.10 & 0.47 & .003 & $* *$ \\
\hline 25 & 9.00 & 0.47 & 8.74 & 0.61 & 0.26 & 0.08 & 0.44 & .005 & ** \\
\hline 26 & 10.95 & 0.49 & 10.63 & 0.64 & 0.32 & 0.13 & 0.51 & .001 & $* * *$ \\
\hline 27 & 10.35 & 0.73 & 10.06 & 0.72 & 0.29 & 0.04 & 0.53 & .021 & $*$ \\
\hline 31 & 5.44 & 0.44 & 5.19 & 0.47 & 0.25 & 0.10 & 0.40 & .001 & $* * *$ \\
\hline 32 & 5.55 & 0.47 & 5.35 & 0.59 & 0.21 & 0.03 & 0.39 & .024 & $*$ \\
\hline 33 & 6.75 & 0.58 & 6.42 & 0.53 & 0.33 & 0.15 & 0.52 & .001 & $* * *$ \\
\hline 34 & 7.44 & 0.49 & 7.23 & 0.49 & 0.21 & 0.05 & 0.38 & .012 & $*$ \\
\hline 35 & 7.97 & 0.46 & 7.89 & 0.60 & 0.08 & -0.10 & 0.26 & .373 & \\
\hline 36 & 10.30 & 0.55 & 10.04 & 0.50 & 0.25 & 0.08 & 0.43 & .005 & $* *$ \\
\hline 37 & 9.80 & 0.56 & 9.46 & 0.67 & 0.34 & 0.14 & 0.55 & .001 & $* * *$ \\
\hline 41 & 5.43 & 0.43 & 5.19 & 0.48 & 0.25 & 0.09 & 0.40 & .002 & $* *$ \\
\hline 42 & 5.54 & 0.48 & 5.33 & 0.57 & 0.21 & 0.03 & 0.38 & .021 & $*$ \\
\hline 43 & 6.73 & 0.55 & 6.44 & 0.48 & 0.29 & 0.11 & 0.46 & .001 & $* * *$ \\
\hline 44 & 7.42 & 0.48 & 7.24 & 0.51 & 0.18 & 0.01 & 0.35 & .036 & $*$ \\
\hline 45 & 7.98 & 0.48 & 7.86 & 0.55 & 0.12 & -0.05 & 0.30 & .158 & \\
\hline 46 & 10.28 & 0.55 & 10.09 & 0.44 & 0.20 & 0.03 & 0.37 & .024 & $*$ \\
\hline 47 & 9.62 & 1.13 & 9.46 & 0.67 & 0.16 & -0.16 & 0.48 & .317 & \\
\hline
\end{tabular}

*FDI notation; DC, digital caliper; CI, confidence interval; SD, standard deviation; Mean diff., mean differences; (***pd" 0.001$)$, $(* * p d " 0.01)$ and (*pd" 0.05). 
J.Hard Tissue Biology Vol. 24(2):155 -168, 2015

Table 8. Maxillary teeth diagonal crown diameters measurement via DC, Norms and Inter sex disparities.

\begin{tabular}{|c|c|c|c|c|c|c|c|c|c|}
\hline Variables* & Male & & Femal & & Mean diff. & $95 \%$ CI & & $P$ & \\
\hline & Mean & SD & Mean & SD & & Lower & Upper & & \\
\hline 11 MxDDP & 8.36 & 0.44 & 8.03 & 0.50 & 0.33 & 0.17 & 0.48 & .001 & *** \\
\hline 11MxDMP & 8.38 & 0.50 & 8.04 & 0.45 & 0.34 & 0.18 & 0.50 & .001 & $* * *$ \\
\hline 12 MxDDP & 6.74 & 0.46 & 6.61 & 0.53 & 0.13 & -0.04 & 0.29 & .134 & \\
\hline 12 MxDMP & 6.67 & 0.53 & 6.54 & 0.55 & 0.14 & -0.05 & 0.32 & .143 & \\
\hline 13MxDDP & 7.66 & 0.37 & 7.35 & 0.41 & 0.31 & 0.18 & 0.44 & .001 & $* * *$ \\
\hline 13МxDMP & 7.83 & 0.43 & 7.51 & 0.46 & 0.32 & 0.17 & 0.47 & .001 & $* * *$ \\
\hline 14 MxDDP & 8.35 & 0.57 & 8.09 & 0.55 & 0.25 & 0.06 & 0.44 & .009 & $* *$ \\
\hline 14 MxDMP & 8.18 & 0.51 & 7.86 & 0.52 & 0.32 & 0.14 & 0.49 & .001 & $* * *$ \\
\hline 15 MxDDP & 8.29 & 0.52 & 8.00 & 0.62 & 0.29 & 0.09 & 0.48 & .004 & $* *$ \\
\hline 15 MxDMP & 8.31 & 0.52 & 7.95 & 0.59 & 0.36 & 0.17 & 0.54 & .001 & $* * *$ \\
\hline 16 MxDDP & 11.01 & 0.57 & 10.72 & 0.61 & 0.29 & 0.09 & 0.49 & .005 & $* *$ \\
\hline 16 MxDMP & 12.45 & 0.62 & 12.17 & 0.57 & 0.28 & 0.08 & 0.48 & .007 & $* *$ \\
\hline 17 MxDDP & 9.58 & 0.85 & 9.63 & 0.79 & -0.05 & -0.33 & 0.23 & .712 & \\
\hline 17MxDMP & 10.86 & 1.04 & 10.78 & 0.91 & 0.08 & -0.25 & 0.41 & .644 & \\
\hline 21 MxDDP & 8.31 & 0.56 & 8.03 & 0.50 & 0.29 & 0.11 & 0.47 & .002 & ** \\
\hline 21 MxDMP & 8.33 & 0.63 & 8.04 & 0.45 & 0.29 & 0.10 & 0.48 & .003 & $* *$ \\
\hline 22 MxDDP & 6.74 & 0.46 & 6.61 & 0.53 & 0.13 & -0.04 & 0.29 & .137 & \\
\hline 22 MxDMP & 6.66 & 0.54 & 6.54 & 0.55 & 0.12 & -0.06 & 0.31 & .182 & \\
\hline 23 MxDDP & 7.65 & 0.39 & 7.35 & 0.41 & 0.30 & 0.16 & 0.43 & .001 & $* * *$ \\
\hline 23 MxDMP & 7.82 & 0.44 & 7.51 & 0.46 & 0.31 & 0.16 & 0.46 & .001 & $* * *$ \\
\hline 24 MxDDP & 8.33 & 0.61 & 8.09 & 0.55 & 0.23 & 0.04 & 0.43 & .020 & ** \\
\hline 24 MxDMP & 8.16 & 0.51 & 7.86 & 0.52 & 0.30 & 0.12 & 0.47 & .001 & $* * *$ \\
\hline 25 MxDDP & 8.28 & 0.53 & 8.00 & 0.62 & 0.28 & 0.09 & 0.48 & .005 & $* *$ \\
\hline 25 MxDMP & 8.29 & 0.52 & 7.95 & 0.59 & 0.34 & 0.15 & 0.53 & .001 & $* * *$ \\
\hline 26 MxDDP & 11.01 & 0.57 & 10.72 & 0.61 & 0.29 & 0.09 & 0.49 & .005 & $* *$ \\
\hline 26MxDMP & 12.44 & 0.62 & 12.17 & 0.57 & 0.27 & 0.07 & 0.48 & .009 & $* *$ \\
\hline 27 MxDDP & 9.60 & 0.85 & 9.63 & 0.79 & -0.03 & -0.31 & 0.25 & .842 & \\
\hline
\end{tabular}

*FDI notation; DC, digital caliper; MxDDP, maxillary diagonal distopalatal diameter; MxDMP, maxillary diagonal mesiopalatal diameter; CI, confidence interval; SD, standard deviation; Mean diff., mean differences; (***pd" 0.001), (**pd" 0.01) and (*pd" 0.05).

Table 9. Mandibular teeth diagonal crown diameters measurement via DC Norms and Inter sex disparities.

\begin{tabular}{|c|c|c|c|c|c|c|c|c|c|}
\hline Variables* & Male & & Femal & & Mean diff. & $95 \%$ CI & & $P$ & \\
\hline & Mean & SD & Mean & SD & & Lower & Upper & & \\
\hline 31 MnDDL & 5.31 & 0.35 & 5.20 & 0.55 & 0.11 & -0.04 & 0.26 & .160 & \\
\hline 31 MnDML & 5.21 & 0.34 & 5.12 & 0.55 & 0.10 & -0.06 & 0.25 & .217 & \\
\hline 32 MnDDL & 5.81 & 0.40 & 5.66 & 0.46 & 0.15 & 0.01 & 0.30 & .038 & \\
\hline 32 MnDML & 5.75 & 0.41 & 5.62 & 0.48 & 0.13 & -0.02 & 0.28 & .095 & \\
\hline 33 MnDDL & 6.65 & 0.36 & 6.38 & 0.50 & 0.27 & 0.13 & 0.42 & .001 & $* * *$ \\
\hline 33 MnDML & 6.88 & 0.40 & 6.60 & 0.48 & 0.28 & 0.14 & 0.43 & .001 & **** \\
\hline 34 MnDDL & 6.93 & 0.39 & 6.80 & 0.46 & 0.13 & -0.01 & 0.27 & .079 & \\
\hline 34 MnDML & 7.30 & 0.43 & 7.04 & 0.51 & 0.26 & 0.10 & 0.42 & .002 & $* *$ \\
\hline 35 MnDDL & 7.60 & 0.46 & 7.43 & 0.55 & 0.17 & 0.00 & 0.34 & .048 & * \\
\hline 35 MnDML & 7.83 & 0.50 & 7.50 & 0.64 & 0.34 & 0.14 & 0.53 & .001 & $* * *$ \\
\hline 36 MnDDL & 11.39 & 0.57 & 11.06 & 0.60 & 0.33 & 0.13 & 0.52 & .001 & $* * *$ \\
\hline $36 \mathrm{MnDML}$ & 11.85 & 0.57 & 11.51 & 0.59 & 0.34 & 0.15 & 0.54 & .001 & $* * *$ \\
\hline 37 MnDDL & 10.90 & 0.70 & 10.43 & 0.67 & 0.47 & 0.24 & 0.70 & .001 & $* * *$ \\
\hline 37 MnDML & 10.97 & 0.65 & 10.66 & 0.65 & 0.31 & 0.09 & 0.53 & .006 & $* *$ \\
\hline 41 MnDDL & 5.31 & 0.35 & 5.20 & 0.54 & 0.11 & -0.04 & 0.26 & .162 & \\
\hline 41 MnDML & 5.21 & 0.34 & 5.11 & 0.55 & 0.10 & -0.06 & 0.25 & .215 & \\
\hline 42 MnDDL & 5.81 & 0.40 & 5.66 & 0.46 & 0.15 & 0.01 & 0.30 & .037 & $*$ \\
\hline 42 MnDML & 5.75 & 0.41 & 5.62 & 0.48 & 0.13 & -0.02 & 0.28 & .093 & \\
\hline 43 MnDDL & 6.65 & 0.36 & 6.38 & 0.50 & 0.28 & 0.13 & 0.42 & .001 & $* * *$ \\
\hline 43 MnDML & 6.88 & 0.40 & 6.60 & 0.48 & 0.28 & 0.13 & 0.43 & .001 & $* * *$ \\
\hline 44 MnDDL & 6.93 & 0.39 & 6.80 & 0.46 & 0.13 & -0.02 & 0.27 & .081 & \\
\hline 44 MnDML & 7.30 & 0.43 & 7.04 & 0.51 & 0.26 & 0.10 & 0.42 & .002 & $* * *$ \\
\hline 45 MnDDL & 7.60 & 0.46 & 7.43 & 0.55 & 0.17 & 0.00 & 0.34 & .048 & $*$ \\
\hline 45 MnDML & 7.83 & 0.50 & 7.50 & 0.64 & 0.34 & 0.14 & 0.53 & .001 & *** \\
\hline 46 MnDDL & 11.39 & 0.57 & 11.06 & 0.60 & 0.33 & 0.13 & 0.52 & .001 & $* * *$ \\
\hline 46 MnDML & 11.96 & 1.09 & 11.51 & 0.59 & 0.45 & 0.15 & 0.75 & .004 & $* *$ \\
\hline 47 MnDDL & 10.90 & 0.70 & 10.43 & 0.67 & 0.47 & 0.24 & 0.70 & .001 & *** \\
\hline
\end{tabular}

*FDI notation; DC, digital caliper; MnDDL, mandibular diagonal distolingual diameter; MnDML, mandibular diagonal distolingual diameter; CI, confidence interval; SD, standard deviation; Mean diff., mean differences; (***pd" 0.001), (**pd"0.01) and (*pd" 0.05$)$. 
Fazal Shahid et al.: Geomorphometrics of Tooth Size and Arch Dimension

Table 10. Maxillary and mandibular arch dimension measurement via DC Norms and Inter sex disparities.

\begin{tabular}{|c|c|c|c|c|c|c|c|c|c|}
\hline \multirow[t]{2}{*}{ Variables } & \multicolumn{2}{|l|}{ Male } & \multicolumn{2}{|l|}{ Female } & \multicolumn{3}{|c|}{ Mean diff. 95\% CI } & \multicolumn{2}{|l|}{$P$} \\
\hline & Mean & SD & Mean & SD & & Lower & Upper & & \\
\hline UAP & 97.52 & 4.54 & 94.75 & 4.08 & 2.77 & 1.31 & 4.23 & .001 & $* * *$ \\
\hline UAL & 130.42 & 4.49 & 127.26 & 5.36 & 3.17 & 1.51 & 4.82 & .001 & $* * *$ \\
\hline UICW & 35.99 & 1.94 & 34.24 & 2.34 & 1.75 & 1.03 & 2.47 & .001 & $* * *$ \\
\hline UI1PW & 43.37 & 2.06 & 41.99 & 3.06 & 1.38 & 0.51 & 2.24 & .002 & ** \\
\hline UI2PW & 48.97 & 2.31 & 47.04 & 2.45 & 1.94 & 1.13 & 2.74 & .001 & $* * *$ \\
\hline UIMW & 53.97 & 2.58 & 52.16 & 2.34 & 1.81 & 0.98 & 2.65 & .001 & $* * *$ \\
\hline LAP & 90.61 & 4.97 & 87.88 & 3.73 & 2.73 & 1.23 & 4.23 & .001 & $* * *$ \\
\hline LAL & 112.94 & 5.65 & 109.99 & 5.12 & 2.95 & 1.12 & 4.78 & .002 & ** \\
\hline LICW & 27.05 & 2.00 & 26.23 & 1.82 & 0.82 & 0.17 & 1.47 & .014 & $*$ \\
\hline LI1PW & 35.88 & 1.94 & 34.49 & 2.53 & 1.40 & 0.64 & 2.15 & .001 & $* * *$ \\
\hline LI2PW & 41.78 & 2.47 & 40.43 & 2.57 & 1.35 & 0.50 & 2.20 & .002 & $* *$ \\
\hline LIMW & 46.70 & 2.44 & 45.15 & 2.36 & 1.54 & 0.73 & 2.35 & .001 & $* * *$ \\
\hline
\end{tabular}

DC, digital caliper; UAP, upper arch perimeter; UAL, upper arch length; UICW, upper inter canine width; UI1PW, upper inter first premolar width; UI2PW, upper inter second premolar width; UIMW, upper inter molar width; LAP, lower arch perimeter; LAL, lower arch length; LICW, Lower inter canine width; LI1PW, Lower inter first premolar width; LI2PW, Lower inter second premolar width; LIMW, Lower inter molar width; CI, confidence interval; SD, standard deviation; Mean diff., mean differences; $(* * *$ pd" 0.001$)$, $(* *$ pd" 0.01) and (*pd" 0.05).

Table 11. Mesiodistal measurement via SM, Norms and Inter sexes disparities.

\begin{tabular}{|c|c|c|c|c|c|c|c|c|c|}
\hline \multicolumn{3}{|c|}{ Variables* Male } & \multicolumn{2}{|c|}{ Female } & \multicolumn{3}{|c|}{ Mean diff. 95\% CI } & \multicolumn{2}{|l|}{$P$} \\
\hline & Mean & SD & Mean & $\mathrm{SD}$ & & Lower & Upper & & \\
\hline 11 & 8.72 & 0.44 & 8.44 & 0.51 & 0.28 & 0.12 & 0.44 & .001 & *** \\
\hline 12 & 6.96 & 0.50 & 6.77 & 0.47 & 0.19 & 0.03 & 0.36 & .022 & $*$ \\
\hline 13 & 7.98 & 0.45 & 7.71 & 0.48 & 0.27 & 0.11 & 0.42 & .001 & *** \\
\hline 14 & 7.05 & 0.45 & 6.86 & 0.41 & 0.18 & 0.04 & 0.33 & .014 & $*$ \\
\hline 15 & 6.76 & 0.46 & 6.65 & 0.49 & 0.11 & -0.05 & 0.27 & .183 & \\
\hline 16 & 10.20 & 0.68 & 9.97 & 0.48 & 0.23 & 0.03 & 0.43 & .027 & $*$ \\
\hline 17 & 9.46 & 0.58 & 9.06 & 0.52 & 0.40 & 0.21 & 0.58 & .001 & *** \\
\hline 21 & 8.73 & 0.45 & 8.37 & 0.50 & 0.35 & 0.19 & 0.51 & .001 & *** \\
\hline 22 & 6.91 & 0.51 & 6.73 & 0.47 & 0.18 & 0.02 & 0.35 & .032 & $*$ \\
\hline 23 & 7.95 & 0.43 & 7.66 & 0.49 & 0.29 & 0.13 & 0.44 & .001 & *** \\
\hline 24 & 7.09 & 0.46 & 6.92 & 0.41 & 0.17 & 0.03 & 0.32 & .022 & $*$ \\
\hline 25 & 6.75 & 0.46 & 6.59 & 0.48 & 0.16 & 0.00 & 0.32 & .046 & * \\
\hline 26 & 10.15 & 0.60 & 9.92 & 0.52 & 0.23 & 0.04 & 0.42 & .018 & * \\
\hline 27 & 9.46 & 0.59 & 9.14 & 0.46 & 0.31 & 0.13 & 0.49 & .001 & *** \\
\hline 31 & 5.52 & 0.34 & 5.49 & 0.39 & 0.03 & -0.10 & 0.15 & .681 & \\
\hline 32 & 6.06 & 0.40 & 5.93 & 0.38 & 0.13 & 0.00 & 0.26 & .056 & $*$ \\
\hline 33 & 7.06 & 0.40 & 6.82 & 0.48 & 0.24 & 0.09 & 0.38 & .002 & $* *$ \\
\hline 34 & 7.05 & 0.44 & 7.00 & 0.40 & 0.05 & -0.09 & 0.20 & .448 & \\
\hline 35 & 7.07 & 0.45 & 6.95 & 0.46 & 0.12 & -0.04 & 0.27 & .138 & \\
\hline 36 & 11.24 & 0.74 & 10.98 & 0.72 & 0.26 & 0.01 & 0.51 & .039 & * \\
\hline 37 & 10.22 & 0.66 & 9.81 & 0.66 & 0.41 & 0.19 & 0.63 & .001 & $* * *$ \\
\hline 41 & 5.51 & 0.34 & 5.45 & 0.40 & 0.06 & -0.06 & 0.18 & .341 & \\
\hline 42 & 6.02 & 0.36 & 5.87 & 0.30 & 0.15 & 0.03 & 0.26 & .011 & $* *$ \\
\hline 43 & 6.99 & 0.42 & 6.67 & 0.85 & 0.32 & 0.10 & 0.54 & .005 & $* *$ \\
\hline 44 & 7.06 & 0.44 & 7.00 & 0.39 & 0.06 & -0.08 & 0.20 & .398 & \\
\hline 45 & 7.04 & 0.47 & 6.92 & 0.51 & 0.11 & -0.05 & 0.28 & .176 & \\
\hline 46 & 11.29 & 0.71 & 10.99 & 0.71 & 0.29 & 0.05 & 0.54 & .017 & $*$ \\
\hline 47 & 10.22 & 0.70 & 9.85 & 0.66 & 0.37 & 0.14 & 0.60 & .002 & $* *$ \\
\hline
\end{tabular}

*FDI notation; SM, hirox digital stereomicroscope; CI, confidence interval; SD, standard deviation; Mean diff., mean differences; (***pd" 0.001), (**pd" 0.01) and (*pd" 0.05).

arch dimension data between DC and SM techniques. There were no significant disparities observed between the both techniques.

\section{Teeth size and arch dimension norms and inter sex disparities}

Tables 6, 7, 8, 9 and 10, and Tables 11, 12, 13, 14 and 15 show tooth size and arch dimensions comparisons between males and females for DC and SM techniques. The developed norms for the mesiodistal, buccolingual, diagonal maxillary, diagonal mandibular and arch dimension respectively with significantly greater values for males in relation to females (*pd" 0.05 to ***pd" $0.001)$. 
J.Hard Tissue Biology Vol. 24(2):155 -168, 2015

Table 12. Buccolingual crown dimension measurement via SM, Norms and Inter sex disparities.

\begin{tabular}{|c|c|c|c|c|c|c|c|c|c|}
\hline Variables* & Male & & Female & & Mean diff. & $95 \%$ CI & & $P$ & \\
\hline & Mean & SD & Mean & SD & & Lower & Upper & & \\
\hline 11 & 6.78 & 0.59 & 6.27 & 0.55 & 0.51 & 0.32 & 0.70 & .001 & $* * *$ \\
\hline 12 & 5.82 & 0.52 & 5.45 & 0.55 & 0.37 & 0.19 & 0.55 & .001 & $* * *$ \\
\hline 13 & 7.75 & 0.60 & 7.33 & 0.50 & 0.42 & 0.23 & 0.60 & .001 & *** \\
\hline 14 & 8.94 & 0.52 & 8.65 & 0.52 & 0.29 & 0.11 & 0.47 & .001 & $* * *$ \\
\hline 15 & 9.02 & 0.49 & 8.75 & 0.59 & 0.27 & 0.09 & 0.45 & .004 & $* *$ \\
\hline 16 & 10.96 & 0.51 & 10.64 & 0.62 & 0.32 & 0.13 & 0.51 & .001 & $* * *$ \\
\hline 17 & 10.36 & 0.65 & 10.30 & 0.76 & 0.07 & -0.17 & 0.30 & .587 & \\
\hline 21 & 6.79 & 0.57 & 6.28 & 0.54 & 0.50 & 0.32 & 0.69 & .001 & $* * *$ \\
\hline 22 & 5.80 & 0.55 & 5.47 & 0.51 & 0.33 & 0.15 & 0.51 & .001 & $* * *$ \\
\hline 23 & 7.74 & 0.55 & 7.29 & 0.55 & 0.45 & 0.27 & 0.64 & .001 & $* * *$ \\
\hline 24 & 8.92 & 0.55 & 8.68 & 0.53 & 0.24 & 0.06 & 0.42 & .011 & $*$ \\
\hline 25 & 9.01 & 0.47 & 8.76 & 0.60 & 0.25 & 0.07 & 0.43 & .008 & $* *$ \\
\hline 26 & 10.96 & 0.49 & 10.64 & 0.63 & 0.31 & 0.12 & 0.50 & .001 & $* * *$ \\
\hline 27 & 10.34 & 0.71 & 10.17 & 0.71 & 0.16 & -0.08 & 0.41 & .179 & \\
\hline 31 & 5.45 & 0.43 & 5.16 & 0.47 & 0.29 & 0.14 & 0.44 & .001 & $* * *$ \\
\hline 32 & 5.56 & 0.47 & 5.36 & 0.59 & 0.20 & 0.02 & 0.38 & .029 & $*$ \\
\hline 33 & 6.76 & 0.58 & 6.38 & 0.54 & 0.38 & 0.19 & 0.57 & .001 & $* * *$ \\
\hline 34 & 7.44 & 0.50 & 7.22 & 0.50 & 0.22 & 0.05 & 0.39 & .010 & $* *$ \\
\hline 35 & 7.98 & 0.46 & 7.83 & 0.63 & 0.15 & -0.03 & 0.34 & .105 & \\
\hline 36 & 10.30 & 0.55 & 10.04 & 0.51 & 0.26 & 0.08 & 0.45 & .004 & ** \\
\hline 37 & 9.81 & 0.56 & 9.50 & 0.64 & 0.31 & 0.11 & 0.52 & .002 & $* *$ \\
\hline 41 & 5.44 & 0.43 & 5.15 & 0.48 & 0.29 & 0.13 & 0.44 & .001 & *** \\
\hline 42 & 5.55 & 0.48 & 5.34 & 0.57 & 0.21 & 0.03 & 0.38 & .021 & $* *$ \\
\hline 43 & 6.73 & 0.55 & 6.40 & 0.49 & 0.33 & 0.16 & 0.51 & .001 & *** \\
\hline 44 & 7.43 & 0.48 & 7.24 & 0.52 & 0.19 & 0.02 & 0.36 & .029 & $*$ \\
\hline 45 & 7.99 & 0.48 & 7.80 & 0.58 & 0.19 & 0.01 & 0.37 & .038 & $*$ \\
\hline 46 & 10.29 & 0.55 & 10.08 & 0.46 & 0.21 & 0.04 & 0.38 & .017 & $*$ \\
\hline 47 & 9.75 & 0.51 & 9.55 & 0.48 & 0.21 & 0.04 & 0.38 & .016 & $* *$ \\
\hline
\end{tabular}

*FDI notation; SM, hirox digital stereomicroscope; CI, confidence interval; SD, standard deviation; Mean diff., mean differences; $(* * *$ pd" 0.001), (**pd" 0.01) and (*pd" 0.05).

\section{Teeth size comparison sides disparities for the maxillary and} mandibular teeth.

Disparities between the right and left side for the mesiodistal, buccolingual, diagonal maxillary and mandibular teeth size respectively. Most of the variables demonstrated no significant differences, except for the few variables (Maxillary lateral incisor [0.047], canine [0.026] and first premolar [0.01] and Mandibular lateral incisor [0.008]) of the mesiodistal tooth size (*pd" 0.05 to **pd" 0.01).

\section{Discussion}

Teeth size has been extensively deliberated by means of ordinary morphometric technique. Progresses in digital acquisitions and scanning have relieved the route to captivate such measurements, and have furthermore made it promising to record the location of landmarks as structured. Some scholars have used these thoughts to study teeth size in two-dimensional ( $x, y)$ directs from a photographic acquisition ${ }^{23,24)}$. Benefits of geomorphometrics have been utilized to evaluate and look out the effect of different types of mechanics on the treatment of class II division 1 malocclusion ${ }^{25,26)}$. Size and shape of human molars were also investigated, analyzed and compared via traditional and innovative geomorphometrics techniques; in addition to that the dental arch shapes were also investigated by the same techniques ${ }^{26,27}$.

Several studies in relation to teeth size; mesiodistal ${ }^{28-31)}$ buccolingual $^{32)}$ and arch dimension ${ }^{28,33,34)}$ has been done on the Pakistani population by sliding caliper but no one used the diagonal teeth dimension and digital models for such investigations ${ }^{28-31)}$. The current study demonstrated the mesiodistal, buccolingual, diagonal maxillary, diagonal mandibular and arch dimension via conventional DC and for the very first time through a new SM digital models technique.

There were no significant differences observed in the data acquisition via both suggested methods. Our study provided the odontometric norms for teeth size and arch dimension of the maxilla and mandible. Teeth size and arch dimension also showed sexual disparities with the males showed the greater values for all measurements. The current study revealed significant sexual disparities for the mesiodistal tooth width are in accordance with other studies ${ }^{35-37)}$. However, some other studies have reported no sexual disparities for mesiodistal tooth width ${ }^{38-41)}$.

In relation to mesiodistal and buccolingual crown diameters, current study showed sexual disparities, which is consistent with published studies for various ethnic groups ${ }^{9,11,42-45,46)}$. Current study showed norms for diagonal crown diameters where male teeth were larger than female. Our results are in agreement with various 
Fazal Shahid et al.: Geomorphometrics of Tooth Size and Arch Dimension

Table 13. Maxillary arch diagonal crown dimension measurement via SM, Norms and Inter sex disparities.

\begin{tabular}{|c|c|c|c|c|c|c|c|c|c|}
\hline \multirow{2}{*}{ Variables* } & \multicolumn{2}{|l|}{ Male } & \multicolumn{2}{|c|}{ Female } & \multicolumn{3}{|c|}{ Mean diff. 95\% CI } & \multicolumn{2}{|l|}{$P$} \\
\hline & Mean & SD & Mean & SD & & Lower & Upper & & \\
\hline 11 MxDDP & 8.36 & 0.44 & 8.06 & 0.50 & 0.30 & 0.14 & 0.46 & .001 & $* * *$ \\
\hline 11MxDMP & 8.39 & 0.50 & 8.07 & 0.45 & 0.31 & 0.15 & 0.47 & .001 & $* * *$ \\
\hline 12 MxDDP & 6.74 & 0.46 & 6.61 & 0.53 & 0.13 & -0.04 & 0.30 & .122 & \\
\hline 12 MxDMP & 6.68 & 0.53 & 6.57 & 0.53 & 0.11 & -0.07 & 0.29 & .225 & \\
\hline 13MxDDP & 7.66 & 0.37 & 7.40 & 0.38 & 0.26 & 0.14 & 0.39 & .001 & $* * *$ \\
\hline 13МxDMP & 7.84 & 0.43 & 7.55 & 0.48 & 0.28 & 0.13 & 0.44 & .001 & $* * *$ \\
\hline 14 MxDDP & 8.35 & 0.57 & 8.12 & 0.55 & 0.23 & 0.04 & 0.42 & .018 & $* *$ \\
\hline 14 MxDMP & 8.18 & 0.51 & 7.84 & 0.52 & 0.35 & 0.17 & 0.52 & .001 & $* * *$ \\
\hline 15 MxDDP & 8.29 & 0.52 & 8.04 & 0.62 & 0.25 & 0.06 & 0.45 & .010 & $* *$ \\
\hline 15 MxDMP & 8.31 & 0.52 & 7.94 & 0.59 & 0.37 & 0.19 & 0.56 & .001 & $* * *$ \\
\hline 16 MxDDP & 11.01 & 0.58 & 10.73 & 0.61 & 0.28 & 0.08 & 0.48 & .006 & $* *$ \\
\hline 16 MxDMP & 12.41 & 0.68 & 12.17 & 0.57 & 0.25 & 0.03 & 0.46 & .025 & $* *$ \\
\hline 17 MxDDP & 9.59 & 0.85 & 9.69 & 0.78 & -0.10 & -0.38 & 0.18 & .469 & \\
\hline 17MXDMP & 10.87 & 1.04 & 10.78 & 0.90 & 0.08 & -0.25 & 0.41 & .618 & \\
\hline 21 MxDDP & 8.32 & 0.56 & 8.06 & 0.50 & 0.26 & 0.08 & 0.44 & .005 & ** \\
\hline 21 MxDMP & 8.34 & 0.63 & 8.07 & 0.45 & 0.26 & 0.08 & 0.45 & .006 & ** \\
\hline 22 MxDDP & 6.74 & 0.46 & 6.61 & 0.53 & 0.13 & -0.04 & 0.30 & .123 & \\
\hline 22 MxDMP & 6.67 & 0.54 & 6.57 & 0.53 & 0.10 & -0.08 & 0.28 & .272 & \\
\hline 23 MxDDP & 7.65 & 0.39 & 7.40 & 0.38 & 0.25 & 0.12 & 0.38 & .001 & $* * *$ \\
\hline 23 MxDMP & 7.83 & 0.44 & 7.56 & 0.48 & 0.27 & 0.12 & 0.43 & .001 & $* * *$ \\
\hline 24 MxDDP & 8.33 & 0.61 & 8.12 & 0.55 & 0.21 & 0.01 & 0.41 & .037 & $*$ \\
\hline 24 MxDMP & 8.16 & 0.51 & 7.83 & 0.52 & 0.33 & 0.15 & 0.50 & .001 & $* * *$ \\
\hline 25 MxDDP & 8.29 & 0.53 & 8.04 & 0.63 & 0.25 & 0.05 & 0.44 & .014 & $* *$ \\
\hline 25 MxDMP & 8.29 & 0.52 & 7.94 & 0.59 & 0.36 & 0.17 & 0.54 & .001 & $* * *$ \\
\hline 26 MxDDP & 11.01 & 0.58 & 10.73 & 0.61 & 0.28 & 0.08 & 0.48 & .006 & $* *$ \\
\hline 26MXDMP & 12.44 & 0.63 & 12.17 & 0.57 & 0.28 & 0.07 & 0.48 & .008 & $* *$ \\
\hline 27 MxDDP & 9.61 & 0.85 & 9.59 & 1.26 & 0.01 & -0.34 & 0.37 & .938 & \\
\hline 27 MxDMP & 10.86 & 1.03 & 10.78 & 0.90 & 0.07 & -0.26 & 0.40 & .659 & \\
\hline
\end{tabular}

*FDI notation; SM, hirox digital stereomicroscope; MxDDP, maxillary diagonal distopalatal diameter; MxDMP, maxillary diagonal mesiopalatal diameter; CI, confidence interval; SD, standard deviation; Mean diff., mean differences; (***pd" 0.001), (**pd" 0.01) and (*pd" 0.05).

studies formerly carried out by means of odontometric measurements with the avowal of sexual disparities ${ }^{11,13,47-49)}$. Arch dimensions of the maxilla and mandible were also measured. The arch dimensions showed sexual disparities, with the male having the larger arch dimension than in female like other investigator ${ }^{50)}$.

Side differences were reported by various researchers for maxillary and mandibular arches ${ }^{31,51-52)}$. However others found no significant side differences in tooth size comparison ${ }^{19,28,53)}$. For the comparison of the right and left side maxillary and mandibular teeth size, we observed the asymmetries only in the mesiodistal width of the lateral incisors, canine and first premolar in maxilla and lateral incisor in mandible .

SM repossession is reckless and effectual because the digital models can be stored according to subject numeric data. An addition, it is possible to view digital models at numerous whereabouts linked to internet $\left.{ }^{18,20,54}\right)$, SM allowing patients to be treated at multiple locations with calm admittance of clinician and researcher to their records. The digital data for dental model can be warehoused on an organization CPU's hard drive, on movable flash drive and CDs. These new digital models untangle many snags come across with conventional plaster study models Recent state-of-the-art innovations have enhanced the process of cast fabrication and manipulation ${ }^{55}$.

In comparison to SM the DC has the shortcoming like, time consumption, difficulty in placement of device by operator, very prone to error and also the damage to the dental models due to its sharp beaks were common. The same time the measurement via DC is manual, cause the corporeal mutilation and create dust or extra muddle.

Our study provides a database of tooth size and arch dimensions from both measurement techniques for first time on Pakistani population. These norms will be helpful for the clinical treatment planning in orthodontic, prosthodontics, and restorative dentistry. Contemporaneously the norms will be of great value in forensic dentistry, and dental anthropology.

In conclusion, there were no significant differences observed in the data acquisition via conventional DC and SM.

- Norms were developed based on DC and SM, the teeth size were quantified in-

$>$ Mesiodistal crown diameter.

$>$ Buccolingual crown diameter.

$>$ Diagonal crown diameter.

- Norms were developed for the arch dimension of maxilla and mandible based on DC and SM techniques.

- There is no difference between the right and left side of the maxillary and mandibular teeth except for the few variables in mesiodistal crown diameters.

- Mesiodistal, buccolingual, diagonal teeth crown diameters and 165 
J.Hard Tissue Biology Vol. 24(2):155 -168, 2015

Table 14. Mandibular arch diagonal crown dimension measurement via SM, Norms and Inter sex disparities.

\begin{tabular}{|c|c|c|c|c|c|c|c|c|c|}
\hline \multirow{2}{*}{ Variables* } & \multicolumn{2}{|l|}{ Male } & \multicolumn{2}{|c|}{ Female } & \multicolumn{3}{|c|}{ Mean diff. 95\% CI } & \multicolumn{2}{|l|}{$\boldsymbol{P}$} \\
\hline & Mean & SD & Mean & SD & & Lower & Upper & & \\
\hline 31 MnDDL & 5.31 & 0.35 & 5.28 & 0.52 & 0.03 & -0.11 & 0.18 & .668 & \\
\hline 31 MnDML & 5.22 & 0.34 & 5.21 & 0.55 & 0.01 & -0.14 & 0.16 & .903 & \\
\hline 32 MnDDL & 5.82 & 0.40 & 5.70 & 0.46 & 0.12 & -0.02 & 0.26 & .104 & \\
\hline 32 MnDML & 5.75 & 0.41 & 5.68 & 0.48 & 0.08 & -0.07 & 0.23 & .310 & \\
\hline 33 MnDDL & 6.66 & 0.36 & 6.44 & 0.47 & 0.21 & 0.08 & 0.35 & .003 & $* *$ \\
\hline 33 MnDML & 6.89 & 0.40 & 6.62 & 0.47 & 0.27 & 0.12 & 0.41 & .001 & $* * *$ \\
\hline 34 MnDDL & 6.93 & 0.40 & 6.82 & 0.46 & 0.11 & -0.03 & 0.25 & .130 & \\
\hline 34 MnDML & 7.30 & 0.44 & 7.07 & 0.50 & 0.23 & 0.08 & 0.39 & .004 & ** \\
\hline 35 MnDDL & 7.61 & 0.46 & 7.50 & 0.58 & 0.11 & -0.07 & 0.29 & .220 & \\
\hline 35 MnDML & 7.84 & 0.50 & 7.62 & 0.65 & 0.22 & 0.02 & 0.41 & .028 & * \\
\hline 36 MnDDL & 11.39 & 0.57 & 11.10 & 0.59 & 0.29 & 0.09 & 0.48 & .004 & ** \\
\hline $36 \mathrm{MnDML}$ & 11.86 & 0.57 & 11.58 & 0.57 & 0.28 & 0.09 & 0.47 & .004 & ** \\
\hline 37 MnDDL & 10.91 & 0.70 & 10.51 & 0.67 & 0.40 & 0.17 & 0.63 & .001 & $* * *$ \\
\hline 37 MnDML & 10.99 & 0.66 & 10.70 & 0.67 & 0.28 & 0.06 & 0.51 & .013 & $*$ \\
\hline 41 MnDDL & 5.31 & 0.35 & 5.28 & 0.52 & 0.03 & -0.12 & 0.18 & .685 & \\
\hline 41 MnDML & 5.22 & 0.34 & 5.21 & 0.55 & 0.01 & -0.14 & 0.16 & .920 & \\
\hline 42 MnDDL & 5.82 & 0.40 & 5.70 & 0.46 & 0.12 & -0.02 & 0.27 & .101 & \\
\hline 42 MnDML & 5.75 & 0.41 & 5.68 & 0.48 & 0.08 & -0.07 & 0.23 & .304 & \\
\hline 43 MnDDL & 6.66 & 0.36 & 6.44 & 0.47 & 0.21 & 0.07 & 0.35 & .003 & $* *$ \\
\hline $43 \mathrm{MnDML}$ & 6.89 & 0.40 & 6.62 & 0.47 & 0.27 & 0.12 & 0.42 & .001 & $* * *$ \\
\hline 44 MnDDL & 6.93 & 0.40 & 6.82 & 0.46 & 0.11 & -0.03 & 0.26 & .124 & \\
\hline 44 MnDML & 7.30 & 0.44 & 7.07 & 0.50 & 0.24 & 0.08 & 0.39 & .004 & $* *$ \\
\hline 45 MnDDL & 7.61 & 0.46 & 7.50 & 0.58 & 0.11 & -0.06 & 0.29 & .211 & \\
\hline 45 MnDML & 7.84 & 0.51 & 7.62 & 0.65 & 0.22 & 0.03 & 0.42 & .026 & \\
\hline 46 MnDDL & 11.39 & 0.57 & 11.09 & 0.59 & 0.30 & 0.11 & 0.50 & .003 & $* *$ \\
\hline $46 \mathrm{MnDML}$ & 11.86 & 0.57 & 11.58 & 0.57 & 0.28 & 0.09 & 0.47 & .004 & $* *$ \\
\hline 47 MnDDL & 10.91 & 0.70 & 10.49 & 0.67 & 0.42 & 0.19 & 0.65 & .001 & $* * *$ \\
\hline 47 MnDML & 10.98 & 0.65 & 10.70 & 0.67 & 0.28 & 0.05 & 0.50 & .015 & * \\
\hline
\end{tabular}

*FDI notation; SM, hirox digital stereomicroscope; MnDDL, mandibular diagonal distolingual diameter; MnDML, mandibular diagonal mesiolingual diameter; CI, confidence interval; SD, standard deviation; Mean diff., mean differences; $\left({ }^{* * *}\right.$ pd" 0.001$),(* * p d " 0.01)$ and (*pd" 0.05).

Table 15. Maxillary and mandibular arch dimension measurement via SM, Norms and Inter sex disparities.

\begin{tabular}{|c|c|c|c|c|c|c|c|c|c|}
\hline \multirow[t]{2}{*}{ Variables } & \multicolumn{2}{|l|}{ Male } & \multicolumn{2}{|l|}{ Female } & \multicolumn{3}{|c|}{ Mean diff. 95\% CI } & \multicolumn{2}{|l|}{$P$} \\
\hline & Mean & SD & Mean & SD & & Lower & Upper & & \\
\hline UAP & 97.51 & 4.55 & 94.87 & 4.40 & 2.64 & 1.13 & 4.15 & .001 & $* * *$ \\
\hline UAL & 130.56 & 4.75 & 127.39 & 5.65 & 3.17 & 1.42 & 4.92 & .001 & $* * *$ \\
\hline UICW & 35.99 & 1.94 & 34.26 & 2.35 & 1.73 & 1.00 & 2.45 & .001 & $* * *$ \\
\hline UI1PW & 43.37 & 2.06 & 42.00 & 3.06 & 1.37 & 0.51 & 2.24 & .002 & $* *$ \\
\hline UI2PW & 48.97 & 2.31 & 47.04 & 2.45 & 1.93 & 1.13 & 2.74 & .001 & $* * *$ \\
\hline UIMW & 53.97 & 2.58 & 52.15 & 2.34 & 1.82 & 0.98 & 2.65 & .001 & $* * *$ \\
\hline LAP & 90.71 & 4.97 & 87.86 & 3.73 & 2.85 & 1.35 & 4.35 & .001 & $* * *$ \\
\hline LAL & 111.53 & 4.21 & 109.84 & 4.93 & 1.69 & 0.15 & 3.23 & .032 & $*$ \\
\hline LICW & 27.05 & 2.00 & 26.24 & 1.82 & 0.82 & 0.17 & 1.47 & .014 & * \\
\hline LI1PW & 35.88 & 1.94 & 34.49 & 2.53 & 1.40 & 0.64 & 2.15 & .001 & $* * *$ \\
\hline LI2PW & 41.78 & 2.47 & 40.43 & 2.57 & 1.35 & 0.50 & 2.20 & .002 & $* *$ \\
\hline LIMW & 46.70 & 2.44 & 45.16 & 2.36 & 1.55 & 0.73 & 2.36 & .001 & *** \\
\hline
\end{tabular}

SM, hirox digital stereomicroscope; UAP, upper arch perimeter; UAL, upper arch length; UICW, upper inter canine width; UI1PW, upper inter first premolar width; UI2PW, upper inter second premolar width; UIMW, upper inter molar width; LAP, lower arch perimeter; LAL, lower arch length; LICW, Lower inter canine width; LI1 PW, Lower inter first premolar width; LI2PW, Lower inter second premolar width; LIMW, Lower inter molar width; CI, confidence interval; SD, standard deviation; Mean diff., mean differences; (***pd" 0.001), (**pd" 0.01) and (*pd"0.05).

arch dimension presented the sexual dimorphism with the greatest value for male were observed.

- The developed norms will be help-full adjunct in orthodontic treatment planning for; tooth size discrepancies, tooth size and arch length discrepancies.
Acknowledgement

Universiti Sains Malaysia, short-term grant 304/PPSG/ 61313104.

\section{References}


Fazal Shahid et al.: Geomorphometrics of Tooth Size and Arch Dimension

1. Bishara SE, Khadivi P and Jakobsen JR. Changes in tooth size arch length relationships from the deciduous to the permanent dentition: A longitudinal study. Am J Orthod Dentofacial Orthop 108: 607-613, 1995

2. Bolton WA. Disharmony In Tooth Size And Its Relation To The Analysis And Treatment Of Malocclusion. Angle Orthod 28: 113-130, 1958

3. Bolton WA. The clinical application of a tooth-size analysis. Am J Orthod 48: 504-529, 1962

4. Alam MK and Iida J. Overjet, overbite and dental midline shift as predictors of tooth size discrepancy in a Bangladeshi population and a graphical overview of global tooth size ratios. Acta Odontol Scand 71: 1520-1531, 2013

5. Acharya $\mathrm{AB}$ and Mainali S. Sex discrimination potential of buccolingual and mesiodistal tooth dimensions. J Forensic Sci 53: 790-792, 2008

6. Yuen KK, So LL and Tang EL. Mesiodistal crown diameters of the primary and permanent teeth in southern Chinese a longitudinal study. Eur J Orthod 19: 721-731, 1997

7. Ling JY and Wong RW. Tooth dimensions of Southern Chinese. Homo 58: 67-73, 2007

8. Lund $\mathrm{H}$ and Mörnstad H. Gender determination by odontometrics in a Swedish population. J Forensic Odontostomatol 17: 30-34, 1999

9. Iscan MY and Kedici PS. Sexual variation in bucco-lingual dimensions in Turkish dentition. Forensic Sci Int 137: 160164, 2003

10. Acharya $\mathrm{AB}$ and Mainali S. Univariate sex dimorphism in the Nepalese dentition and the use of discriminant functions in gender assessment. Forensic Sci Int 173: 47-56, 2007

11. Khamis MF, Taylor JA, Malik SN and Townsend GC. Odontometric sex variation in Malaysians with application to sex prediction. Forensic Sci Int 234: 183 e1-183e7, 2014

12. Ateş M, Karaman F, Işcan MY and Erdem TL. Sexual differences in Turkish dentition. Legal Med 8: 288-292, 2006

13. Karaman F. Use of Diagonal Teeth Measurements in Predicting Gender in a Turkish Population. J Forensic Sci 51: 630-635, 2006

14. Zelditch ML, Swiderski DL and Sheets HD. Geometric morphometrics for biologists: a primer. Academic Press : 437-441, 2012

15. Vandenbroucke JP, Von Elm E, Altman D G, Gøtzsche PC, Mulrow CD, Pocock SJ, and Egger M. Strengthening the Reporting of Observational Studies in Epidemiology (STROBE): explanation and elaboration. Ann Intern Med 147: 163-194, 2007

16. Prabhu $\mathrm{S}$ and Acharya $\mathrm{AB}$. Odontometric sex assessment in Indians. Forensic Sci Int 192: 129 e1-129e5, 2009

17. Dupont WD and Plummer WD Jr. Power and sample size calculations for studies involving linear regression. Control
Clin Trials 19: 589-601, 1998

18. Shahid F, Alam MK, Khamis MF, Muraoka R, Nakano K and Okafuji N. Validity and Reliability of digital model measurements: A digital stereomicroscopic study. J Hard Tissue Biol 23: 439-444, 2014

19. Al- Khatib AR, Rajion ZA, Masudi SaM, Hassan R, Anderson PJ and Townsend GC. Tooth size and dental arch dimensions: a stereophotogrammetric study in Southeast Asian Malays. Orthod Craniofac Res 14: 243-253, 2011

20. Alam MK, Shahid F, Kathiravan P, Basaruddin A and Khamis, MF. Bolton tooth size ratio and its relation with arch widths, arch length and arch perimeter: A cone beam computed tomography (CBCT) study. Acta Odont Scand 72: 10471053, 2014

21. Lavelle C. Comparison of the deciduous teeth between Caucasoid, Negroid, and Mongoloid population samples. Dent Pract Dent Rec 21: 121-124, 1970

22. Houston WJ. The analysis of errors in orthodontic measurements. Am J Orthod 83: 382-390, 1983

23. Robinson DL, Blackwell PG, Stillman EC and Brook AH. Planar Procrustes analysis of tooth shape. Arch Oral Biol 46: 191-199, 2001

24. Robinson DL, Blackwell PG, Stillman EC and Brook AH. Impact of landmark reliability on the planar Procrustes analysis of tooth shape. Arch Oral Biol 47: 545-554, 2002

25. Singh GD. Morphospatial analysis of soft- tissue profile in patients with Class II division 1 malocclusion treated using twin block appliances: geometric morphometrics. Orthod Craniofac Res 5: 38-50, 2002

26. Singh GD and Thind BS. Effects of the headgear- activator Teuscher appliance in the treatment of class II division 1 malocclusion: a geometric morphometric study. Orthod Craniofac Res 6: 88-95, 2003

27. Bernal V. Size and shape analysis of human molars: comparing traditional and geometric morphometric techniques. Homo 58: 279-296, 2007

28. Adnani IQ, Shahid F, Sana Z, Fahad A and Misbha S. Application of Moyer's prediction table in a sample of Karachi population. Pak Orthod J 3: 16-20, 2011

29. Batool I, Abbas A, Rizvi S and Abbas I. Evaluation of tooth size discrepancy in different malocclusion groups. J Ayub Med Coll Abbottabad 20: 51-54, 2008

30. Memon S and Fida M. Comparison of three mixed dentition analysis methods in orthodontic patients at AKUH. J Coll Physicians Surg Pak 20: 533-537, 2010

31. Bherwani AK and Fida M. Development of a prediction equation for the mixed dentition in a Pakistani sample. Am J Orthod Dentofacial Orthop 140: 626-632, 2011

32. Rehmani SN and Fida M. Assessment of fluctuating asymmetry in permanent dentition. Pak Orthod J 4: 39-43, 
2012

33. Tajik I, Mushtaq N and Khan M. Arch Forms Among Different Angle Classifications A Study. Pak Oral Den J 31: 92-95, 2011

34. Hamid MW and Babar MI. Dental crowding and its relationship to tooth size and arch dimensions. Pak Oral Dent J 25: 47-52, 2005

35. Smith SS, Buschang PH and Watanabe E. Interarch tooth size relationships of 3 populations: "Does Bolton's analysis apply?”. Am J Orthod Dentofacial Orthop 117: 169-174, 2000

36. Munjal S, Duggal R, Kahlon SS and Bansal S. Tooth size discrepancies in individuals presenting with different malocclusions. Ind J Dental Sci 2: 15-17, 2010

37. Fattahi HR, Pakshir HR and Hedayati Z. Comparison of tooth size discrepancies among different malocclusion groups. Eur J Orthod 28: 491-495, 2006

38. Sharma R, Kumar S and Singla A. Prevalence of tooth size discrepancy among North Indian orthodontic patients. Contemp Clin Dent 2: 170-175, 2011

39. Doodamani GM, Khala AS, and Mala Manohar U. Assessment of crown angulations, crown inclinations, and tooth size discrepancies in a South Indian population. Contemp Clin Dent 2: 176-181, 2011

40. Akyalçin S, Dogan S, Dinçer B, Erdinc AM and Oncað G. Bolton tooth size discrepancies in skeletal class I individuals presenting with different dental Angle classifications. Angle Orthod 76: 637-643, 2006

41. Crosby DR and Alexander CG. The occurrence of tooth size discrepancies among different malocclusion groups. Am J Orthod Dentofacial Orthop 95: 457-461, 1989

42. Garn SM, Lewis AB and Kerewsky RS. Sexual dimorphism in the buccolingual tooth diameter. J Dent Res 45: 1819, 1966

43. Lakhanpal M GN, Rao NC and Vashisth S. Tooth Dimension Variations as a Gender Determinant in Permanent Maxillary
Teeth. JSM Dent 2: 1-5, 2013

44. Doris JM, Bernard BW, Kuftinec MM and Stom D. A biometric study of tooth size and dental crowding. Am J Orthod 79: 326-336, 1981

45. Townsend GC and Brown T. Tooth size characteristics of Australian Aborigines. Occas Pap Hum Biol 1: 17-38, 1979

46. Alam MK, Hassan R, Mahmood Z and Haq ME. Determination and comparison of tooth size and tooth size ration in normal occlusion and different malocclusion groups. Int Med J 20: 462-465, 2013

47. Garn SM, Lewis AB, Swindler DR and Kerewsky RS. Genetic control of sexual dimorphism in tooth size. J Dent Res 46: 963-972, 1967

48. Anderson DL and Thompson GW. Interrelationships and sex differences of dental and skeletal measurements. J Dent Res 52: 431-438, 1973

49. Kaur S and Chattopadhyay PK. Sexual dimorphism of incisors: a study of the Jat Sikhs. Leg Med 5: 261-262, 2003

50. Quraishi BA, Hussain SS and Alvi AR. Comparison of arch width dimensions among class I and class II division 1 malocclusion groups. J Pak Dent Assoc 21: 5-11, 2012

51. Kieser JA and Groeneveld HT. Fluctuating odontometric asymmetry in an urban South African black population. J Dent Res 67: 1200-1205, 1988

52. Kieser JA, Groeneveld HT and Preston CB. Fluctuating odontometric asymmetry in the Lengua Indians of Paraguay. Ann Hum Biol 13: 489-498, 1986

53. Alam MK, Shahid F, Purmal K, Ahmad B and Khamis MF. Tooth size and dental arch dimension measurement through Cone beam Tomography: effect of age and gender. Res J Rec Sci 3: 85-94, 2014

54. Redmond WR, Redmond WJ and Redmond MJ. Clinical implications of digital orthodontics. Am J Orthod Dentofacial Orthop 117: 240-241, 2000

55. Peluso MJ, Josell SD, Levine SW and Lorei BJ. Digital models: An introduction. Semi Orthod 10: 226-238, 2004 